\title{
Preface: Internet multimedia computing and service
}

\author{
Shuqiang Jiang • Changsheng Xu • Yong Rui • \\ Alberto Del Bimbo • Hongxun Yao
}

Published online: 1 November 2012

(C) Springer Science+Business Media New York 2012

There exist large amounts of multimedia content on the Internet. However, current consumer expectations for multimedia applications still far exceed the achievement of today's state of the art technologies. There is a technical gap between rich multimedia contents and multimedia computing technologies. This special issue focuses on the problem of Internet Multimedia Computing and Service, which aims at providing readers new perspectives on this hot research area. This special issue contains 10 papers covering various aspects of Internet multimedia, which can be categorized into three groups: semantic multimedia understanding, adaptive Internet multimedia access, and human-centered multimedia service. We believe that the papers included in this special issue not only provide outstanding research contributions but also form the latest overview of research interest in this field. The key ideas and contributions of these papers are summarized as below.

\section{Semantic multimedia understanding}

Semantically understanding of multimedia data is an essential problem for Internet multimedia applications, where feature representation plays an important role. The paper

\author{
S. Jiang $(\bowtie)$ \\ Institute of Comp. Tech., Chinese Academy of Sciences, Beijing, China \\ e-mail: sqjiang@ict.ac.cn \\ C. $\mathrm{Xu}$ \\ Institute of Automation, Chinese Academy of Sciences, Beijing, China \\ e-mail: csxu@nlpr.ia.ac.cn \\ Y. Rui \\ Microsoft, Beijing, China \\ e-mail: yongrui@microsoft.com
}

A. Del Bimbo

University of Firenze, Firenze, Italy

e-mail: delbimbo@dsi.unifi.it

H. Yao

Harbin Institute of Technology, Harbin, China

e-mail: h.yao@hit.edu.cn 
"Evaluation of local features and classifiers in BOW model for image classification" by QuWu-Liu-Xie-Wang (doi: 10.1007/s11042-012-1107-z) studies the popular BOW image representation model and evaluates the performance of different detectors and classifiers for image classification.

To obtain fine-grained image annotation results at the region level, the paper "Region level annotation by fuzzy based contextual cueing label propagation by Zhong-Liu-LiuChung (doi: 10.1007/s11042-011-0954-3) proposes a label to region assignment (LRA) method called Fuzzy-based contextual-cueing label propagation (FCLP). Contextual cueing means the visual elements with their surroundings. This paper is one of the first attempts to integrate contextual cueing in the multimedia computing domain.

The paper "Typicality ranking: beyond accuracy for video semantic annotation" by TangHua (doi: 10.1007/s11042-011-0892-0) deals with video annotation from the point-view of typical relevance. The proposed method relaxes the semantic labels of training data to quantitatively typicality scores. Moreover, a new evaluation criterion compatible with the proposed method is also proposed.

Contextual information in the Internet is a useful clue to better understand and utilize multimedia data. The paper "Integrating bilingual search results for automatic junk image filtering" by Yang-Peng-Feng-Pan (doi: 10.1007/s11042-012-1051-y) proposes an interesting idea by using bilingual image retrieval results to remove the junk images and obtain more satisfactory retrieval results.

\section{Adaptive Internet multimedia access}

Multimedia data are accessed by users with different devices and network conditions. The following three papers work on adaptive Internet multimedia access tying to provide users more flexible solutions to access multimedia data.

The paper "Seamless video access for mobile devices by content-aware utility-based adaptation" by Nur-Kodikara Arachchi-Dogan-Kondoz (doi: 10.1007/s11042-012-1120-2) proposes an adaptive decision making method to decide suitable scalable parameters for adaptive video access using a utility based approach.

The paper "Semantic aware sport image resizing jointly using seam carving and warping" by Wu-Gong-Yuan-Zhang-Cao (doi: 10.1007/s11042-012-1002-7) researches on the problem of determining suitable image sizes for suitable devices based on the content analysis of sport images. The method in this paper is composed of two steps. The first is court field detection and court boundary segmentation. The second step implements warping and seam carving to generate the final results.

Super-resolution is a special kind of image resizing and is a hot research topic recently. The paper "Image super-resolution based on multi-space sparse representation" by Jing-ShiKong-Ding-Yin (doi: 10.1007/s11042-011-0953-4) proposes a super-resolution method by decomposing images into structural and textual component and reconstructing them based on the technique of multi-space sparse representation.

\section{Human-centered multimedia service}

Human is the final user of multimedia data. How to satisfy user's requirement from various aspects is also an important research problem. The paper "A three-level framework for affective content analysis and its case studies" by Xu-Wang-He-Jin-Luo-Lu 
(doi: 10.1007/s11042-012-1046-8) investigates the problem of video content affective analysis by introducing a mid-level representation, which includes audio and textual semantic information.

To generate attractive and interesting human representation, 3D face based techniques are useful solutions in many Internet application scenarios. The paper "Expansion of 3D face sample set based on genetic algorithm" by Ge-Yin-Sun-Jing (doi: 10.1007/s11042-012-1102-4) proposes a method to automatically expand 3D face training samples to enrich the 3D face database.

Recommendation is an effective technique of personalized multimedia service. The paper "Interactive ads recommendation with contextual search on product topic space" by WangWang-Duan-Tian-Lu (doi: 10.1007/s11042-011-0866-2) proposes an advertisement recommendation service system by considering hierarchical concept relations and contextual search information. Users can interactively select their favorite key frames, and users' intentions can be included in the system.

We are grateful to all authors for their contributions to this special issue. We appreciate the reviewers for their valuable comments to ensure the quality of all the accepted papers. We would also like to thank Editor-in-Chief Prof. Borko Furht for approving this special issue. Finally, our special thanks go to Maria Mhanilet de Leon, Laura A. Lander, Melissa Fearon, and Razel Gerona at the Editorial Office for supporting and organizing the review process. We hope that these articles will stimulate further research activities for this promising research area.

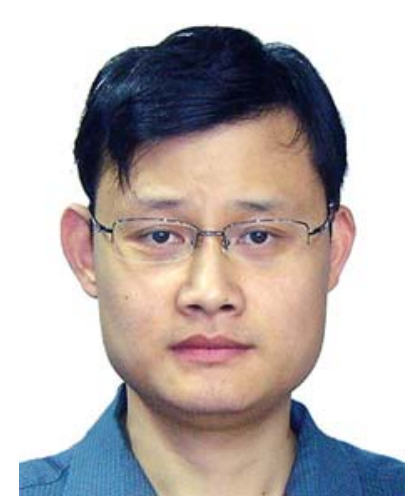

Shuqiang Jiang is an associate professor with the Institute of Computing Technology, Chinese Academy of Sciences, Beijing. He is also with the Key Laboratory of Intelligent Information Processing, Chinese Academy of Sciences. He research interests include multimedia processing and semantic understanding, pattern recognition, and computer vision. He has authored or coauthored more than 100 papers on the related research topics. Dr. Jiang was supported by the New-Star program of Science and Technology of Beijing Metropolis in 2008. He won the Lu Jiaxi Young Talent Award from Chinese Academy of Sciences in 2012, and the CCF Award of Science and Technology in 2012. $\mathrm{He}$ is the senior member of IEEE, member of ACM, CCF, and YOCSEF. Dr. Jiang is the executive committee member of ACM SIGMM China chapter. He has been serving as the guest editor of the special issues for PR and MTA. He is the program chair of ICIMCS2010, special session chair of PCM2008, ICIMCS2012, area chair of PCIVT2011, publicity chair of PCM2011 and proceedings chair of MMSP2011. He has also served as a TPC member for more than 20 well-known conferences, including ACM Multimedia, CVPR, ICCV, ICME, ICIP, and PCM. 


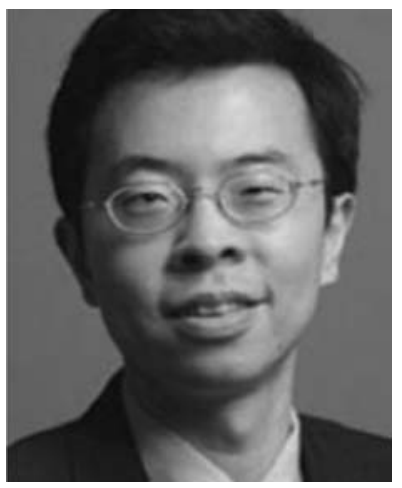

Changsheng $\mathbf{X u}$ is a professor in National Lab of Pattern Recognition, Institute of Automation, Chinese Academy of Sciences and Executive Director of China-Singapore Institute of Digital Media. His research interests include multimedia content analysis/indexing/retrieval, pattern recognition and computer vision. He has hold 30 granted/pending patents and published over 200 refereed research papers in these areas. Dr. Xu is an Associate Editor of ACM Transactions on Multimedia Computing, Communications and Applications and ACM/Springer Multimedia Systems Journal. He served as Program Chair of ACM Multimedia 2009. He has served as associate editor, guest editor, general chair, program chair, area/track chair, special session organizer, session chair and TPC member for over 20 IEEE and ACM prestigious multimedia journals, conferences and workshops.

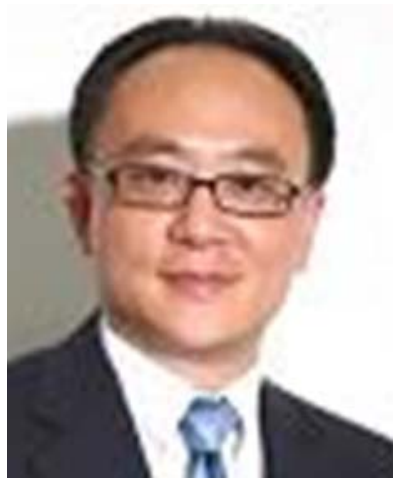

Yong Rui is a Senior Director at Microsoft Research Asia. From 2010 to 2012, he was a Senior Director and General Manager of Microsoft Asia-Pacific R\&D (ARD) Group where he is in charge of China Innovation. From 2008 to 2010, he was the Director responsible for Microsoft Education Product in China. From 2006 to 2008, he was ARD's first Director of R\&D Strategy, and from 1999 to 2006, he was leading the Multimedia Collaboration group at Microsoft Research, Redmond, USA. A Fellow of IEEE, IAPR and SPIE, and a Distinguished Scientist of ACM, Rui is recognized as a pioneer and a world-class expert in Web multimedia search. He holds 40+ US and international patents. He has published 16 books and book chapters, and 100+ referred journal and conference papers. Rui's publications are among the most cited - his top 10 papers have been cited 6,000+ times. Dr. Rui is the Associate Editor-in-Chief of IEEE Multimedia Magazine, an Associate Editor of ACM Trans. on Multimedia Computing, Communication and Applications (TOMCCAP) and a founding editor of International Journal of Multimedia Information Retrieval. He was an Associate Editor of IEEE Trans. on Multimedia (2004-2008), IEEE Trans. on Circuits and Systems for Video Technologies (2006-2010), ACM/Springer Multimedia Systems Journal (2004-2006), and International Journal of Multimedia Tools and Applications (2004-2006). He also serves on the Advisory Board of IEEE Trans. on Automation Science and Engineering. Dr. Rui is on Organizing Committees and Program Committees of 
numerous conferences including ACM Multimedia, IEEE CVPR, IEEE ECCV, IEEE ACCV, IEEE ICIP, IEEE ICASSP, IEEE ICME, SPIE ITCom, ICPR, CIVR, among others. He is General Co-Chair of Conference on Image and Video Retrieval (CIVR) 2006, ACM Multimedia 2009, and ICIMCS 2010, and Program CoChair of ACM Multimedia 2006, Pacific Rim Multimedia (PCM) 2006, and IEEE ICME 2009. Dr. Rui is on the Steering Committees of IEEE ICME, ACM Multimedia, ACM ICMR and PCM. He is the Chair of ACM SIG Multimedia Beijing Chapter. He served as a member of review panels for the US National Science Foundation (NSF), the Chinese National Science Foundation (CNSF), the Australian Research Council and the Research Grants Council of Hong Kong. Dr. Rui received his BS from Southeast University, his MS from Tsinghua University, and his $\mathrm{PhD}$ from University of Illinois at Urbana-Champaign (UIUC). He also holds a Microsoft Leadership Training Certificate from Wharton Business School, University of Pennsylvania.

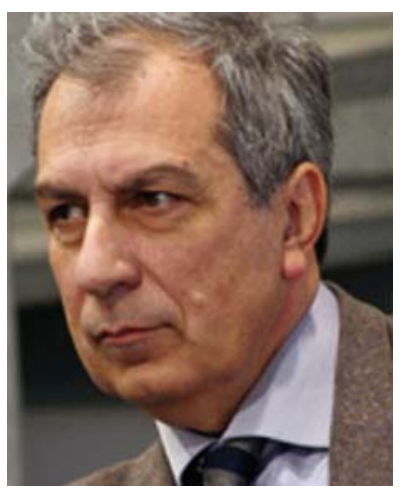

Alberto Del Bimbo is Professor of Computer Engineering at the Faculty of Engineering, University of Firenze. His research interests address analysis and interpretation of images and video and their applications, with particular interest at content-based retrieval in visual and multimedia digital archives, advanced video surveillance and target tracking, and natural man-machine interaction assisted by computer vision.

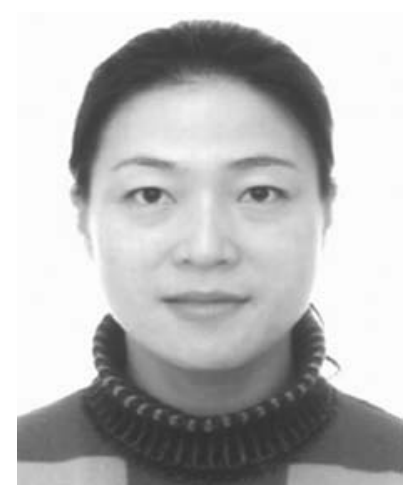

Hongxun Yao received the B.S. and M.S. degrees in computer science from the Harbin Shipbuilding Engineering Institute, Harbin, China, in 1987 and in 1990, respectively, and received Ph.D. degree in computer science from Harbin Institute of Technology in 2003. Currently, she is a professor with School of Computer Science and Technology, Harbin Institute of Technology. Her research interests include computer vision, pattern recognition, multimedia computing, human-computer interaction technology. She has 5 books and over 200 scientific papers published, and won both the honor title of "the new century excellent talent" in China and "enjoy special government allowances expert" in Heilongjiang Province, China. 